\title{
Cardiac striatin interacts with caveolin-3 and calmodulin in a calcium sensitive manner and regulates cardiomyocyte spontaneous contraction rate.
}

\begin{tabular}{|c|c|}
\hline Journal: & Canadian Journal of Physiology and Pharmacology \\
\hline Manuscript ID & cjpp-2017-0155.R2 \\
\hline Manuscript Type: & Article \\
\hline Date Submitted by the Author: & 23-Jul-2017 \\
\hline Complete List of Authors: & $\begin{array}{l}\text { Nader, Moni; Alfaisal University, Physiological Sciences; King Faisal } \\
\text { Specialist Hospital and Research Center, Genetics } \\
\text { Alotaibi, Shahd; Alfaisal University, Physiological Sciences; King Faisal } \\
\text { Specialist Hospital and Research Center, Genetics } \\
\text { Alsolme, Ebtehal; Alfaisal University, Physiological Sciences; King Faisal } \\
\text { Specialist Hospital and Research Center, Genetics } \\
\text { Khalil, Bariaa; Alfaisal University, Physiological Sciences; King Faisal } \\
\text { Specialist Hospital and Research Center, Department of Genetics, } \\
\text { Abu-Zaid, Ahmed; Alfaisal University, Physiological Sciences; King Faisal } \\
\text { Specialist Hospital and Research Center, Department of Genetics, } \\
\text { Alsomali, Rahmah; King Faisal Specialist Hospital and Research Center, } \\
\text { Department of Genetics, } \\
\text { Bakheet, Dana; King Faisal Specialist Hospital and Research Center, } \\
\text { Department of Genetics, } \\
\text { Dzimiri, Nduna; King Faisal Specialist Hospital and Research Center, } \\
\text { Department of Genetics, }\end{array}$ \\
\hline $\begin{array}{l}\text { Is the invited manuscript for } \\
\text { consideration in a Special } \\
\text { Issue?: }\end{array}$ & IACS Sherbrooke 2016 special issue Part 1 \\
\hline Keyword: & Striatin, Contraction rate, Cardiomyocyte, Calmodulin, Caveolin-3 \\
\hline
\end{tabular}

\section{SCHOLARONE" \\ Manuscripts}


Cardiac striatin interacts with caveolin-3 and calmodulin in a calcium sensitive manner and regulates cardiomyocyte spontaneous contraction rate.

Moni Nader ${ }^{1,2}$, Shahd Alotaibi ${ }^{1,2}$, Ebtehal Alsolme ${ }^{1,2}$, Bariaa Khali1 ${ }^{1,2}$, Ahmed AbuZaid $^{1,2}$, Rahmah Alsomali $^{2}$, Dana Bakheet ${ }^{2}$ and Nduna Dzimiri ${ }^{2}$.

${ }^{1}$ Department of Physiological Sciences, College of Medicine, Alfaisal University, Riyadh 11533, Kingdom of Saudi Arabia.

${ }^{2}$ Department of Genetics, King Faisal Specialist Hospital and Research Centre, Riyadh, Kingdom of Saudi Arabia.

Address correspondence to:

Moni Nader, PhD

Department of Physiological Sciences

College of Medicine, Alfaisal University

E-mail: $\underline{\text { mnader@alfaisal.edu }}$

Tel: +966112157674

Fax: +966112157651 


\begin{abstract}
Impaired cardiomyocyte contraction rate is detrimental to cardiac function and often lethal. Despite the advancement in the field, there is a paucity of information regarding the coordination of molecules implicated in regulating the heart rate. Striatin (STRN) is a dynamic protein with binding domains to calmodulin (CaM) and caveolin (Cav), both of which are regulators of myocardial function. However, its role in cardiomyocyte contraction is not yet determined. Herein, we show that STRN is expressed in cardiomyocytes and it is more abundant in atrial myocardium than in ventricles. Cardiac expression of STRN (protein and mRNA) was developmentally regulated with the highest expression being at neonatal stage (day-one) and the lowest in adult rats (13 weeks). CaM pull down assay indicated that the interaction of cardiac STRN with CaM and caveolin-3 (Cav-3) was calcium sensitive. Interestingly, the overexpression of STRN induced an increase $(\sim 2$ folds $)$ in the rate of the spontaneous contraction of cultured cardiomyocytes while the knockdown of STRN reduced their contraction rate $(\sim 40 \%)$. The expression level of STRN was inversely proportional to the interaction of Cav-3 with the CaM/STRN complex. Collectively, our data delineate a novel role for STRN in regulating cardiomyocyte spontaneous contraction rate and the dynamics of the STRN/Cav-3/CaM complex.
\end{abstract}

Keywords: striatin, contraction rate, cardiomyocyte, calmodulin, caveolin-3. 


\section{Introduction}

Contractile dysfunction is tightly associated with disrupted sequence of events linking the excitation of cardiomyocytes to intracellular calcium $\left[\mathrm{Ca}^{2+}\right]_{\mathrm{i}}$ and contraction (a process designated the excitation-contraction (E-C) coupling) (Bers 2002). Although the E-C module is constantly regulated by the autonomic system for rapid adjustments of the heart rate (HR) to the body demand, a sustainably elevated HR becomes maladaptive chronically and is often associated with mortality (Benetos et al. 2003; Cohn et al. 1984; Hori and Okamoto 2012; Jouven et al. 2005). Moreover, variations in the basal HR in normal subjects are commonly accepted as predicators of increased risk of cardiac failure (Zhang et al. 2016). Despite the tremendous advances in the field, a preventive strategy avoiding the detrimentally elevated HR remains far from being reached. This owes to the insufficient understanding of the molecular cues underpinning the E-C coupling mechanism and subsequently the rate of cardiomyocyte contraction.

Striatin (STRN) is a multivalent protein that was originally purified from highly active adenylyl cyclase (AC) rich fractions from rat brain synaptosomes, and was found to bind calmodulin $(\mathrm{CaM})$ in a calcium sensitive manner (Bartoli et al. 1998; Castets et al. 1994; Castets et al. 2000). Both CaM and AC are reportedly regulators of the E-C coupling in the heart and modulators of cardiac contractility (Maier and Bers 2002; Tang et al. 2010). Further studies attributed a role for STRN in a calcium signaling complex regulating rat locomotor activity and the growth of dendrites in cultured neuronal cells (Bartoli et al. 1999). Subsequent characterization of STRN revealed binding domains to dynamics molecules such as protein phosphatase 2A (PP2A) (Gordon et al. 2011; 
Moreno et al. 2000), caveolin-1 (Cav-1) (Gaillard et al. 2001) and $\mathrm{G}_{\alpha i}$ (Lu et al. 2004), all of which are regulators of calcium homeostasis and cardiomyocyte contraction (duBell et al. 1996; Feron et al. 1998; Grimm et al. 1998; Kamishima et al. 2007; Kuschel et al. 1999; Schilling et al. 2016; Young et al. 2001). Moreover, the discovery of the striatin-interacting phosphatases and kinases (STRIPAK) complex implicated an interaction between STRN and the sarcolemmal membrane associated protein (SLMAP), a regulator of cardiomyocyte $\left[\mathrm{Ca}^{2+}\right]_{\mathrm{i}}$ (Goudreault et al. 2009). Recently, a deletion in the 3'-UTR untranslated region of the STRN gene was detected in boxer dogs, a model of arrhythmogenic right ventricular cardiomyopathy. This was associated with decreased expression of STRN in cardiac tissues particularly at the desmosomes, suggesting a central role for STRN in maintaining the HR (Meurs et al. 2010).

Despite the structural features of STRN (interaction with PP2A, CaM, caveolin (Cav), $\mathrm{G}_{\alpha \mathrm{i}}$, and SLMAP), as well as its presence in highly active AC rich fractions, its role in cardiomyocyte contraction remains elusive. Herein, we report that STRN is expressed in cardiomyocytes and that its expression is developmentally regulated in the heart. We also found that cardiac STRN associates with the muscle specific caveolin (Cav-3) and CaM in a calcium sensitive manner. The levels of STRN were proportional to the contraction rate of cardiomyocytes but inversely proportional to the association of Cav-3 with STRN/CaM complex. This study provides novel evidence on the molecular regulator of the contraction rate of cardiomyocyte and emerges STRN as a therapeutic target for cardiac contractile dysfunction. 


\section{Materials and methods}

\section{Cardiomyocytes culture}

Animals were cared for in accordance with the Guide for the Care and Use of Laboratory Animals (2011, published by National Academy Press, Washington, DC 20055) and the use of animals was reviewed and approved by the King Faisal Specialist Hospital and Research Centre Research Advisory Council, Animal Care and Use Committee (RAC\# 2100 026). Neonatal ventricular cardiomyocytes were isolated from 1-day-old rats as previously described (Bkaily et al. 2004). In brief, neonatal day-one pups were decapitated and the lower third of the ventricles were harvested, to avoid Sino-atrial node cells, and minced in S-MEM (Minimum Essential Medium, suspension, Cat\# 11380-052, Thermo Fisher Scientific) supplemented with 0.5\% trypsin (27250-018, Thermo Fisher Scientific). Cardiac tissues were digested in a cell dispenser and several rounds of trypsinizations (S-MEM+Trypsin) were performed to yield the maximum amount of cardiomyocytes. The yielded cells were suspended in fresh MEM (Cat\#12561-056, Thermo Fisher Scientific) supplemented with 10\% fetal bovine serum (FBS, Cat\# F4135, sigma Aldrich) and 1\% Penicillin-Streptomycin-Glutamine (PSG, Cat\# 10378-016, Thermo Fisher Scientific). To segregate cardiomyocytes from non-cardiomyocytes, cells were pre-plated for 1 hour and were subsequently transferred to a new petri dish (cardiomyocytes). The remaining adherent cells on the petri dish contained noncardiomyocytes and were supplemented with MEM (10\% FBS). To rule out possible contamination of cardiomyocytes with non-cardiomyocytes, the cardiomyocyte culture was treated with $10 \mu \mathrm{M}$ of the anti-mitotic agent cytosine arabinofuranoside (Cat\# C1768-1G, Sigma Aldrich) for 24 hours. 


\section{Protein extraction and Western blot}

Cardiac ventricles from neonatal (day 1), 2, 4, and 13 week-old rats were minced with fine pair of scissors in ice-cold lysis radioimmunoprecipitation assay buffer (RIPA buffer, Cat\# R0278, Sigma Aldrich) supplemented with complete protease inhibitor cocktail (Cat\# 11836145001, Roche) and were homogenized using an electrical homogenizer (polytron) on ice. Proteins from cultured cardiomyocytes were extracted by scraping cells with ice-cold RIPA buffer and cellular extracts were kept in RIPA buffer on ice for 30 min for complete cell lysis. In both cases (tissues and cells), proteins were cleared by centrifugation at $10,000 \mathrm{~g}$, for $5 \mathrm{~min}$, at $4^{\circ} \mathrm{C}$. Protein concentration was determined using the Pierce ${ }^{\mathrm{TM}}$ BCA protein assay kit (Cat\# 23225, Thermo Fisher Scientific). A total of 20 $\mu \mathrm{g}$ of proteins was size-fractionated on $4-12 \%$ gradient polyacrylamide gel and separated proteins were transferred to a polyvinylidene difluoride (PVDF) membrane. The membrane was blocked with 5\% non-fat dried milk (NFDM) in Tris Buffered Saline with Tween (TBS-T, Cat\# T9039, Sigma Aldrich) for one hour at room temperature (RT) and was subsequently incubated with primary antibodies for STRN (anti-striatin monoclonal antibody, 1:1,000, Cat\# 610383, BD biosciences), Cav-3 (anti-caveolin-3 rabbit polyclonal antibody, 1,1000, Cat\# ab2912, Abcam), and GAPDH (rabbit antiglyceraldehyde 3-phosphate dehydrogenase [anti-GAPDH] antibody, 1:1,000, Cat\# sc25778, Santa Cruz) in TBS-T (1\% NFDM). After three washes with TBS-T (5 min each), the membrane was incubated with either secondary anti-rabbit-HRP (anti-rabbithorseradish peroxidase, Cat\# 7074S, Cell Signaling), or anti-mouse-HRP (Cat\# 7076S, Cell Signaling) at a final dilution of 1:10,000. Following three washes (5 min each), the 
protein bands were visualized using enhanced chemiluminescence (Cat\# RPN2106, Amersham) and the LAS 4000 gel imaging system.

\section{Real Time PCR}

Ribonucleic acid (RNA) was extracted from heart tissues or cultured cells using the Direct-zol RNA extraction kit (Cat\# R2052, Zymo Research) following the manufacturer's protocol. A total of $5 \mu \mathrm{g}$ of messenger RNA (mRNA) was converted to complimentary deoxyribonucleic acid (cDNA) using the Superscript III Reverse Transcriptase (Cat\# 18080093, Thermo Fisher Scientific) according to the manufacturer's protocol. For amplification of the STRN gene using semi-quantitative PCR, forward (5'GAC CAA GCT ATG GAC GAG CAG GCG-3') and reverse (5'-GCG CTC GAG TCA TAC AAA GAC CTT AGC CA-3') primers were designed for the complete open reading frame of rat STRN (Metabion). Amplification of rat heart STRN was performed using the Herculase II Fusion Enzyme (Ca\# 600677, Agilent Technologies) following the manufacturer's protocol and the amplicons were separated on a $1 \%$ agarose gel (Cat\# 16500-100, Thermo fisher Scientific).

For Real Time PCR, primers designed against rat STRN and GAPDH (FAM and VIC labeled, respectively) were purchased from Applied Biosystems (Cat\# 4331182 and 4352338E, respectively). A volume of $1 \mu \mathrm{l}$ of cDNA was used to amplify STRN and GAPDH simultaneously in a CFX96 Real Time PCR (BioRad). Data were presented as the ratio of STRN/GAPDH mRNA expression.

\section{Adenoviral infection and contraction rate assay}


STRN levels were manipulated in cultured cardiomyocytes by transducing adenoviral particles in cultured cardiomyocytes (multiplicity of infection $[\mathrm{MOI}]=100$ ). Viral particles coding for STRN along with the reporter gene red fluorescent protein (RFPSTRN) or for RFP alone (control) were used for the overexpression (OE) of STRN. For knockdown (KD) assays, both adenoviruses producing STRN short hairpin RNA (STRNshRNA) or scrambled shRNA (U6) were also expressing RFP as a reporter gene. Both adenoviral particles were customized and purchased from Vector Biolabs. The use of reporter gene in both $\mathrm{OE}$ and $\mathrm{KD}$ experiments was essential to identify the infected cells and evaluate the viral transduction efficiency under the microscope.

Successful OE or KD was confirmed by Western blot. To determine the rate of spontaneous contraction, live videos of infected cardiomyocytes (showing red fluorescence) were recorded on confocal microscope (Axio Observer.Z1, Zeiss) equipped with a Slide in module CSU-X1" XLmulti S1 unit to keep the cells at $37^{\circ} \mathrm{C}$ and $5 \% \mathrm{CO}_{2}$. The number of spontaneous contractions of ventricular cardiomyocytes was counted over one min to determine their contraction rate.

\section{Immunofluorescence}

Cultured ventricular cardiomyocytes were washed with Phosphate Buffer Saline (PBS 1X, Cat\# p3813, Sigma Aldrich) and fixed with 4\% paraformaldehyde (Cat\#, 158127, Sigma Aldrich) for $30 \mathrm{~min}$. After 3 washes with PBS (1X) for $5 \mathrm{~min}$ each, aldehyde groups were quenched using 0.1 M glycine (G4392, Sigma Aldrich) for $15 \mathrm{~min}$ and fixed cells were washed twice with PBS (1X) for 5 min each. Permeabilization of cell membranes was done by applying $0.1 \%$ triton $X$, in $0.5 \%$ NFDM supplemented with $1 \%$ 
fetal calf serum (FCS, Cat\# C8056, Sigma Aldrich) in PBS (1X) for $30 \mathrm{~min}$ at RT. Permeabilized cells were incubated with mouse anti-STRN monoclonal antibody $(1: 100)$ and rabbit anti- alpha-actinin antibody (1:800) diluted in $0.1 \%$ triton $\mathrm{X}, 1 \%$ NFDM, and $1.4 \%$ NCS in PBS (1X) overnight at $4^{\circ} \mathrm{C}$. Primary antibodies were withdrawn the next day and cells were washed twice with $1 \mathrm{X}$ PBS for 5 min at RT. Secondary antibody Alexa Fluor 488 goat anti-mouse and Alexa Fluor goat anti-mouse 568 (Cat\# A11001 and A11011, respectively, Thermo Fisher Scientific) were diluted $(1: 1,000)$ in the same primary antibody solution and was applied to the cells for $1 \mathrm{hr}$ at RT. Cells were washed twice (1X PBS) and visualized using LSM 510 confocal microscopy (Zeiss). Sections of labeled cells were sampled and images were processed using the LSM Pascal software. The detector gain, laser power, and image-acquiring settings were maintained constant during image acquisition.

\section{Calmodulin pull down assay}

Calmodulin (CaM) Sepharose 4B beads (Cat\# 17-0529-01, GE Healthcare) were equilibrated as per the manufacturer's protocol and were incubated with $1 \mathrm{mg}$ of proteins from heart lysates of 13-week-old rats (for tissues) or from cell proteins (for cultured ventricular cardiomyocytes) for $1 \mathrm{hr}$ at RT. The protein complex was precipitated by centrifugation at maximum speed and the pelleted beads were washed three times with RIPA buffer. The beads (containing the protein complex interacting with $\mathrm{CaM}$ ) were subsequently suspended in loading buffer (1X) and boiled for 5 min to yield the interacting proteins. The beads were centrifuged for 1 min at maximum speed and the 
proteins (supernatant) were size-fractionated on a polyacrylamide gel to assess for the presence of STRN and Cav-3 as described in the Western blot section. 


\section{Results}

\section{Striatin is expressed in cardiomyocytes.}

The expression of STRN in cardiac tissue has been controversial. Therefore, we amplified the STRN gene using PCR and primers designed for the open reading frame of rat STRN. The PCR product showed a band around $2.4 \mathrm{~Kb}$ corresponding to STRN mRNA (Fig. 1A). Since cardiomyocytes occupy only $40 \%$ of cardiac tissues, we sought to verify if STRN is expressed in cardiomyocytes. Therefore, we cultured cardiomyocytes and non-cardiomyocytes from neonatal rat hearts and assessed the expression of STRN using Western blot. The monoclonal antibody for STRN detected a band $\sim 110 \mathrm{kDa}$, corresponding to STRN, in both cardiomyocytes and noncardiomyocytes lysates confirming that cardiomyocytes express STRN (Fig. 1A). The myosin heavy chain (MHC) band appearing only in the cardiomyocytes lysate, along with the smooth muscle actin (SMA) band that was highly expressed in the noncardiomyocytes lysate confirmed the successful isolation of cardiomyocytes from other cells in provenance from neonatal rat hearts (Fig. 1B). Immunofluorescence using the monoclonal anti-STRN antibody on cultured cardiomyocytes revealed a diffuse distribution of STRN within the sarcoplasm, as well as a clear clustering the sarcolemma (arrows in figure 1C) and the nucleus. The alpha-actinin staining was used as a marker of cardiomyocytes.

\section{The expression of cardiac striatin is developmentally regulated.}

Several reports indicate that STRN is expressed in cardiac tissues however, its expression pattern during cardiac development is not yet elucidated. We applied Real Time PCR and 
assessed the mRNA expression of the STRN gene in rat hearts at postnatal-day-one (P1), 2, 4 and 13 weeks $(2 \mathrm{~W}, 4 \mathrm{~W}$, and $13 \mathrm{~W})$ of age. Data in figure $2 \mathrm{~A}$ shows that the highest expression of STRN mRNA is recorded at neonatal stage, while this expression gradually declines throughout development to reach the lowest level of expression at $13 \mathrm{~W}$. The expression pattern of STRN proteins paralleled that of mRNA exemplified in the Western blot bands of figure 2B; the expression of cardiac STRN being the highest in the hearts of neonates and $2 \mathrm{~W}$ while the lowest expression was at 4 and $13 \mathrm{~W}$. Furthermore, we sought to explore whether STRN is equally expressed in atria and ventricles. Western blot analysis showed that both left and right atria (LA and RA, respectively) expressed higher amounts of STRN when compared to left and right ventricles (LV and RV, respectively) of adult hearts (13W old rats). GAPDH antibody was used as a control for equal loading of proteins.

\section{Cardiac striatin interacts with calmodulin and caveolin-3 in a calcium sensitive manner.}

Both Cav and CaM were shown to regulate cardiac myocyte contraction. STRN interacts with CaM and Cav-1 (an ubiquitous isoform of Cav) in a calcium sensitive manner (Castets et al. 2000; Gaillard et al. 2001). We sought to verify whether cardiac STRN interacts with CaM and Cav-3 (the muscle isoform of Cav) and whether this interaction is calcium sensitive. Therefore, CaM pull down assay was performed on heart lysates from $13 \mathrm{~W}$ old rats and data revealed that cardiac STRN interacts with CaM and Cav-3 as shown by the robust bands of both proteins detected in the CaM-beads lane (in the presence of $2 \mathrm{mM} \mathrm{CaCl}_{2}$ ). Interestingly, the chelation of calcium from the preparation 
(using $2 \mathrm{mM}$ ethylene glycol tetraacetic acid [EGTA]) profoundly reduced the STRN/Cav-3/CaM interactions displayed by less STRN and Cav-3 proteins in the CaMbeads lane (Fig. 3). These results suggest that cardiac STRN plays a role in Cav-3 and calmodulin signaling in a calcium dependent fashion.

\section{The spontaneous contraction rate of ventricular cardiomyocytes is controlled by striatin levels.}

Since both Cav-3 and CaM play a central role in regulating calcium homeostasis and cardiomyocytes contraction, we monitored the spontaneous contraction rate in ventricular cardiomyocytes with depleted endogenous STRN using shRNA (STRN-shRNA). Data in figure 4A confirmed a successful KD of STRN gene using adenoviral transduction particles. Surprisingly, KD of STRN reduced the spontaneous contraction rate of cardiomyocytes by almost $40 \%$ when compared with those carrying the scrambled shRNA (Ctrl-shRNA) (Fig. 4B). In contrast, the OE of STRN (Fig. 4C) induced approximately a two-fold increase in the spontaneous contraction rate of cardiomyocytes (Fig. 4D). This data provides a cogent proof that the levels of STRN are critical to maintain proper cardiomyocytes contraction rate.

\section{Striatin modulates the stability of the Cav-3/CaM complex in cardiomyocytes.}

Our data showed a calcium sensitive interaction between the components of STRN/Cav3/CaM complex. In addition, the levels of STRN revealed essential for cardiomyocyte contraction. Since both Cav-3 and CaM are implicated in the contraction of cardiomyocyte, we opted to verify if the alterations in STRN levels (by means of 
overexpression or knockdown) affects the stability of the STRN/Cav-3/CaM complex, in the presence of calcium $\left(2 \mathrm{mM}\right.$ of $\left.\mathrm{CaCl}_{2}\right)$. Data indicated that neither the $\mathrm{KD}$, nor the $\mathrm{OE}$, of STRN affected the endogenous expression of Cav-3 in ventricular cardiomyocytes (Figure 5A and D). In contrast, the degree of association of Cav-3 with CaM was inversely proportional to the expression level of STRN in these cardiomyocytes. In fact, while the OE of STRN largely reduced the interaction between Cav-3 and the STRN/CaM (Figure 5B and C), the KD of STRN showed opposite results (Figure 5E and F). This data suggests that STRN levels regulate the stability of the STRN/Cav-3/CaM complex in cardiomyocytes. 


\section{Discussion}

Our study demonstrates that STRN is expressed in cardiomyocytes and that it contributes to the regulation of spontaneous rate of firing of new-born cultured ventricular cardiomyocytes. This was evidenced by 1) the expression of STRN in enriched cardiomyocyte culture and the developmental regulation of cardiac STRN (mRNA and proteins), 2) the calcium sensitive interaction between STRN, Cav-3 and CaM, 3) the positive chronotropic effect induced by STRN in cardiomyocytes and (4) the altered interaction between Cav-3 and CaM in cardiomyocytes with manipulated levels of STRN.

The expression of STRN in the heart, particularly in ventricular cardiomyocytes, is controversial. Early studies indicated that STRN mRNA transcripts and/or proteins were found in several tissues including the brain, cerebellum, spinal cord, spleen and lungs, but not in the myocardium (Castets et al. 1996; Castets et al. 2000). Recent studies revealed that STRN expression was increased in cardiac lysates of rats with high sodium diet (Ricchiuti et al. 2011), but decreased in the desmosomes of cardiac histological sections of boxer dogs, a model for ARVC (Meurs et al. 2010). Here we showed that STRN mRNA transcripts and proteins are present in rat cardiac lysates and more importantly in isolated cardiomyocytes (confirmed by the abundant expression of MHC and lower expression of SMA). Of interest, the localization of STRN at the sarcolemma of cultured cardiomyocytes (immunofluorescence data) corroborated previous studies reporting STRN at the desmosomes. This provides a cogent proof that STRN is expressed 
in cardiomyocytes and suggests a role for STRN in regulating the E-C coupling in cardiomyocytes.

During cardiac ontogeny, proteins implicated in the maturation of the E-C coupling undergo substantive changes in their expression levels [reviewed in (Wetzel and Klitzner 1996)], and the regulation of calcium homeostasis parallels the spatial and temporal organization of the E-C module (Ziman et al. 2010). This suggests that STRN may be essential for the maturation and organization of the E-C coupling module in ventricular cardiomyocytes since its expression pattern during cardiac development paralleled that of NCX which is involved in the pacing activity of cardiomyocytes (Boerth et al. 1994; Verdonck et al. 2004). In fact, our data showed that overexpression of STRN in ventricular cardiomyocytes increased their spontaneous rate of contraction which may contribute to ventricular fibrillation. However, the KD of STRN exhibited the opposite effect on the rate of contraction. It is worth noting that the SA nodal cells, which exhibit higher spontaneous contraction rate than the rest of the cardiac myocytes (Marvin et al. 1984), were absent from our ventricular cardiomyocyte culture. Nonetheless, the pacing activity of ventricular cardiomyocytes acquired as function of days in culture (4 days in our experimental model) is in line with previously reported studies (Kimura et al. 1998a; Kimura et al. 1998b). A plausible explanation for these observations could account for the fact that the OE of STRN may have turned ventricular cardiomyocytes into SA nodal cells with intrinsic pacing activity. However, since the same ventricular cardiomyocytes (which are not SA nodal cells) showed lower acquired spontaneous rate of contraction when depleted from STRN, this implies that STRN levels are essential for 
maintaining the electrical properties of the sarcolemma and subsequently the rate of spontaneous contractions. These suggest that the increased expression of endogenous STRN in neonatal ventricular cardiomyocytes may account for the higher rate of firing of these recorded in neonates compared to adult rats (Drugge et al. 1985), although, in normal conditions, heart rate is determined by SA nodal cells. Our data provides unequivocal proof that the levels of STRN are essential for maintaining a proper contraction rate of ventricular cardiomyocytes.

The association of STRN with Cav-3 and CaM in a calcium sensitive manner also implies that STRN may be a part of the E-C coupling module. STRN might be playing the role of a molecular switch similarly to CaM kinase and endothelial nitric oxide synthase (eNOS) that are activated by $\mathrm{Ca}^{2+}-\mathrm{CaM}$ to coordinate calcium homeostasis and contraction of the heart (Wang and Abdel-Rahman 2002; Wu et al. 2001). This is also supported by our data showing that STRN OE in ventricular cardiomyocytes augmented their spontaneous rate of contraction, while KD of STRN exhibited the opposite effect on chronotropy. This was associated with inversely proportional interaction between Cav-3 and STRN/CaM complex. The increased and decreased interaction of STRN and Cav-3 with $\mathrm{CaM}$, in response to STRN OE, or $\mathrm{KD}$, respectively (with unchanged expression of Cav-3), implies that abundant amounts of STRN dissociated Cav-3 from the STRN/Cav3/CaM complex. Nonetheless, CaM could bind Cav-3 independently from STRN however, the levels of striatin appear to be critical for this association. Whether STRN exerts its effect directly on the E-C module or whether it regulates the contraction rate via the Cav-3/CaM complex remains to be determined. A plausible explanation for these 
phenotypical changes in contraction rate is that the increased amounts of the dissociated Cav-3 from STRN/CaM complex may induce positive chronotropy in cardiomyocytes. However, it has been reported that the overexpression of Cav-3 results in negative chronotropy in vivo (Schilling et al. 2016). On the other hand, the stabilization of Cav-3 to plasma membrane is critical to maintaining proper cardiac function (Naito et al. 2015). In fact, heart failure is associated with decreased expression of Cav-3 (Feiner et al. 2011). The disruption of caveolae, or the knockdown of Cav-3, reduced L-type calcium channel (LCC) currents in cultured cardiomyocytes (Balijepalli et al. 2006). This suggests that the effect of STRN overexpression on positive chronotropy may be mediated by targeting Cav-3/CaM to the sarcolemma while the opposite phenotype occurs when silencing the STRN gene.

In conclusion, our data established a novel role for cardiac STRN in regulating the spontaneous contraction rate of ventricular cardiomyocytes, possibly via the modulation of the Cav-3/CaM complex. Our findings provide essential information on the regulation of the E-C coupling in cardiomyocyte and crystalizes STRN out as a novel gene for therapeutic strategies and control of detrimental tachycardia. It is also possible that STRN could be important in determining the spontaneous beating, maturation and differentiation of pluripotent stem cells into ventricular cardiomyocytes with SA node functional properties (Liu et al. 2016). This latter should be verified in the future. 


\section{Acknowledgement}

This work was supported by an operating grant to MN from King Abdulaziz City for Science and Technology (KACST \#13BIO-1452-20). 


\section{References}

Balijepalli, R.C., Foell, J.D., Hall, D.D., Hell, J.W., and Kamp, T.J. 2006. Localization of cardiac L-type Ca2+ channels to a caveolar macromolecular signaling complex is required for 32 -adrenergic regulation. Proceedings of the National Academy of Sciences 103(19): 7500-7505. doi: 10.1073/pnas.0503465103.

Bartoli, M., Monneron, A., and Ladant, D. 1998. Interaction of Calmodulin with Striatin, a WD-repeat Protein Present in Neuronal Dendritic Spines. Journal of Biological Chemistry 273(35): 22248-22253. doi: 10.1074/jbc.273.35.22248.

Bartoli, M., Ternaux, J.-P., Forni, C., Portalier, P., Salin, P., Amalric, M., and Monneron, A. 1999. Down-regulation of striatin, a neuronal calmodulin-binding protein, impairs rat locomotor activity. Journal of Neurobiology 40(2): 234-243. doi: 10.1002/(SICI)1097-4695(199908)40:2<234::AID-NEU9>3.0.CO;2-T.

Benetos, A., Thomas, F., Bean, K., Albaladejo, P., Palatini, P., and Guize, L. 2003. Resting heart rate in older people: a predictor of survival to age 85. Journal of the American Geriatrics Society 51(2): 284-285. doi: 10.1046/j.15325415.2003.51080.x.

Bers, D.M. 2002. Cardiac excitation-contraction coupling. Nature 415(6868): 198205.

Bkaily, G., Nader, M., Avedanian, L., Jacques, D., Perrault, C., Abdel-Samad, D., D'Orléans-Juste, P., Gobeil, F., and Hazzouri, K.M. 2004. Immunofluorescence revealed the presence of NHE-1 in the nuclear membranes of rat cardiomyocytes and isolated nuclei of human, rabbit, and rat aortic and liver tissues. Canadian Journal of Physiology and Pharmacology 82(8-9): 805-811. doi: 10.1139/y04-119. 
Boerth, S.R., Zimmer, D.B., and Artman, M. 1994. Steady-state mRNA levels of the sarcolemmal $\mathrm{Na}(+)-\mathrm{Ca} 2+$ exchanger peak near birth in developing rabbit and rat hearts. Circulation Research 74(2): 354-359. doi: 10.1161/01.res.74.2.354.

Castets, F., Baillat, G., Mirzoeva, S., Mabrouk, K., Garin, J., d'Alayer, J., and Monneron, A. 1994. A Brain Synaptosomal Adenylyl Cyclase of High Specific Activity Is Photolabeled with Azido-ATP. Biochemistry 33(17): 5063-5069. doi: 10.1021/bi00183a009.

Castets, F., Bartoli, M., Barnier, J.V., Baillat, G., Salin, P., Moqrich, A., Bourgeois, J.P., Denizot, F., Rougon, G., Calothy, G., and Monneron, A. 1996. A novel calmodulinbinding protein, belonging to the WD-repeat family, is localized in dendrites of a subset of CNS neurons. The Journal of Cell Biology 134(4): 1051-1062. doi: 10.1083/jcb.134.4.1051.

Castets, F., Rakitina, T., Gaillard, S., Moqrich, A., Mattei, M.G., and Monneron, A. 2000. Zinedin, SG2NA, and striatin are calmodulin-binding, WD repeat proteins principally expressed in the brain. J Biol Chem 275. doi: 10.1074/jbc.M909782199.

Cohn , J.N., Levine , T.B., Olivari , M.T., Garberg , V., Lura , D., Francis , G.S., Simon , A.B., and Rector, T. 1984. Plasma Norepinephrine as a Guide to Prognosis in Patients with Chronic Congestive Heart Failure. New England Journal of Medicine 311(13): 819-823. doi: doi:10.1056/NEJM198409273111303.

Drugge, E.D., Rosen, M.R., and Robinson, R.B. 1985. Neuronal regulation of the development of the alpha-adrenergic chronotropic response in the rat heart. Circulation Research 57(3): 415-423. doi: 10.1161/01.res.57.3.415. 
duBell, W.H., Lederer, W.J., and Rogers, T.B. 1996. Dynamic modulation of excitationcontraction coupling by protein phosphatases in rat ventricular myocytes. The Journal of Physiology 493(3): 793-800. doi: 10.1113/jphysiol.1996.sp021423.

Feiner, E.C., Chung, P., Jasmin, J.F., Zhang, J., Whitaker-Menezes, D., Myers, V., Song, J., Feldman, E.W., Funakoshi, H., Degeorge Jr, B.R., Yelamarty, R.V., Koch, W.J., Lisanti, M.P., McTiernan, C.F., Cheung, J.Y., Bristow, M.R., Chan, T.O., and Feldman, A.M. 2011. Left Ventricular Dysfunction in Murine Models of Heart Failure and in Failing Human Heart is Associated With a Selective Decrease in the Expression of Caveolin3. Journal of Cardiac Failure 17(3): 253-263. doi: http://dx.doi.org/10.1016/j.cardfail.2010.10.008.

Feron, O., Dessy, C., Opel, D.J., Arstall, M.A., Kelly, R.A., and Michel, T. 1998. Modulation of the Endothelial Nitric-oxide Synthase-Caveolin Interaction in Cardiac Myocytes: implications for the autonomic reglation of heart rate. Journal of Biological Chemistry 273(46): 30249-30254. doi: 10.1074/jbc.273.46.30249.

Gaillard, S., Bartoli, M., Castets, F., and Monneron, A. 2001. Striatin, a calmodulindependent scaffolding protein, directly binds caveolin-1. FEBS Letters 508(1): 4952. doi: 10.1016/S0014-5793(01)03020-4.

Gordon, J., Hwang, J., Carrier, K.J., Jones, C.A., Kern, Q.L., Moreno, C.S., Karas, R.H., and Pallas, D.C. 2011. Protein phosphatase 2a (PP2A) binds within the oligomerization domain of striatin and regulates the phosphorylation and activation of the mammalian Ste20-Like kinase Mst3. BMC Biochemistry 12(1): 54. doi: 10.1186/1471-2091-12-54. 
Goudreault, M., D'Ambrosio, L.M., Kean, M.J., Mullin, M.J., Larsen, B.G., Sanchez, A., Chaudhry, S., Chen, G.I., Sicheri, F., Nesvizhskii, A.I., Aebersold, R., Raught, B., and Gingras, A.C. 2009. A PP2A phosphatase high density interaction network identifies a novel striatin-interacting phosphatase and kinase complex linked to the cerebral cavernous malformation 3 (CCM3) protein. Mol Cell Proteomics 8. doi: 10.1074/mcp.M800266-MCP200.

Grimm, M., Gsell, S., Mittmann, C., Nose, M., Scholz, H., Weil, J., and Eschenhagen, T. 1998. Inactivation of Gi $\alpha$ Proteins Increases Arrhythmogenic Effects of $\beta$-Adrenergic Stimulation in the Heart. Journal of Molecular and Cellular Cardiology 30(10): 19171928. doi: http://dx.doi.org/10.1006/jmcc.1998.0769.

Hori, M., and Okamoto, H. 2012. Heart rate as a target of treatment of chronic heart failure. Journal of Cardiology 60(2): 86-90. doi: http://dx.doi.org/10.1016/j.jjcc.2012.06.013.

Jouven , X., Empana , J.-P., Schwartz , P.J., Desnos , M., Courbon , D., and Ducimetière , P. 2005. Heart-Rate Profile during Exercise as a Predictor of Sudden Death. New England Journal of Medicine 352(19): 1951-1958. doi: doi:10.1056/NEJMoa043012. Kamishima, T., Burdyga, T., Gallagher, J.A., and Quayle, J.M. 2007. Caveolin-1 and caveolin-3 regulate $\mathrm{Ca} 2+$ homeostasis of single smooth muscle cells from rat cerebral resistance arteries. American Journal of Physiology - Heart and Circulatory Physiology 293(1): H204-H214. doi: 10.1152/ajpheart.00669.2006.

Kimura, H., Takemura, H., Imoto, K., Furukawa, K., Ohshika, H., and Mochizuki, Y. 1998a. Relation between Spontaneous Contraction and Sarcoplasmic Reticulum 
Function in Cultured Neonatal Rat Cardiac Myocytes. Cellular Signalling 10(5): 349354. doi: http://dx.doi.org/10.1016/S0898-6568(97)00134-4.

Kimura, K., Kimura, H., Yokosawa, N., Isogai, H., Isogai, E., Kozaki, S., Miyamoto, A., Nishikawa, T., Ohshika, H., Kubota, T., and Fujii, N. 1998b. Negative Chronotropic Effect of Botulinum Toxin on Neonatal Rat Cardiac Myocytes. Biochemical and Biophysical Research Communications 244(1): 275-279. doi: http://dx.doi.org/10.1006/bbrc.1998.8188.

Kuschel, M., Zhou, Y.-Y., Cheng, H., Zhang, S.-J., Chen, Y., Lakatta, E.G., and Xiao, R.-P. 1999. Gi Protein-mediated Functional Compartmentalization of Cardiac $\beta 2$ Adrenergic Signaling. Journal of Biological Chemistry 274(31): 22048-22052. doi: 10.1074/jbc.274.31.22048.

Liu, J., Laksman, Z., and Backx, P.H. 2016. The electrophysiological development of cardiomyocytes. Advanced Drug Delivery Reviews 96: 253-273. doi: http://dx.doi.org/10.1016/j.addr.2015.12.023.

Lu, Q., Pallas, D.C., Surks, H.K., Baur, W.E., Mendelsohn, M.E., and Karas, R.H. 2004. Striatin assembles a membrane signaling complex necessary for rapid, nongenomic activation of endothelial NO synthase by estrogen receptor $\alpha$. Proceedings of the National Academy of Sciences of the United States of America 101(49): 1712617131. doi: 10.1073/pnas.0407492101.

Maier, L.S., and Bers, D.M. 2002. Calcium, Calmodulin, and Calcium-Calmodulin Kinase II: Heartbeat to Heartbeat and Beyond. Journal of Molecular and Cellular Cardiology 34(8): 919-939. doi: http://dx.doi.org/10.1006/jmcc.2002.2038. 
Marvin, W.J., Chittick, V.L., Rosenthal, J.K., Sandra, A., Atkins, D.L., and Hermsmeyer, K. 1984. The isolated sinoatrial node cell in primary culture from the newborn rat. Circulation Research 55(2): 253-260. doi: 10.1161/01.res.55.2.253.

Meurs, K.M., Mauceli, E., Lahmers, S., Acland, G.M., White, S.N., and Lindblad-Toh, K. 2010. Genome-wide association identifies a deletion in the 3 ' untranslated region of striatin in a canine model of arrhythmogenic right ventricular cardiomyopathy. Hum Genet 128. doi: 10.1007/s00439-010-0855-y.

Moreno, C.S., Park, S., Nelson, K., Ashby, D., Hubalek, F., Lane, W.S., and Pallas, D.C. 2000. WD40 Repeat Proteins Striatin and S/G2 Nuclear Autoantigen Are Members of a Novel Family of Calmodulin-binding Proteins That Associate with Protein Phosphatase 2A. Journal of Biological Chemistry 275(8): 5257-5263. doi: 10.1074/jbc.275.8.5257.

Naito, D., Ogata, T., Hamaoka, T., Nakanishi, N., Miyagawa, K., Maruyama, N., Kasahara, T., Taniguchi, T., Nishi, M., Matoba, S., and Ueyama, T. 2015. The coiled-coil domain of MURC/cavin-4 is involved in membrane trafficking of caveolin-3 in cardiomyocytes. American Journal of Physiology - Heart and Circulatory Physiology 309(12): H2127-H2136. doi: 10.1152/ajpheart.00446.2015.

Ricchiuti, V., Lapointe, N., Pojoga, L., Yao, T., Tran, L., Williams, G.H., and Adler, G.K. 2011. Dietary sodium intake regulates angiotensin II type 1, mineralocorticoid receptor, and associated signaling proteins in heart. Journal of Endocrinology 211(1): 47-54. doi: 10.1530/joe-10-0458.

Schilling, J.M., Horikawa, Y.T., Zemljic-Harpf, A.E., Vincent, K.P., Tyan, L., Yu, J.K., McCulloch, A.D., Balijepalli, R.C., Patel, H.H., and Roth, D.M. 2016. Electrophysiology 
and metabolism of caveolin-3-overexpressing mice. Basic Research in Cardiology 111(3): 28. doi: 10.1007/s00395-016-0542-9.

Tang, T., Lai, N.C., Hammond, H.K., Roth, D.M., Yang, Y., Guo, T., and Gao, M.H. 2010. Adenylyl Cyclase 6 Deletion Reduces Left Ventricular Hypertrophy, Dilation, Dysfunction, and Fibrosis in Pressure-Overloaded Female Mice. Journal of the American College of Cardiology 55(14): 1476-1486. doi: http://dx.doi.org/10.1016/j.jacc.2009.11.066.

Verdonck, F., Mubagwa, K., and Sipido, K.R. 2004. [Na+] in the subsarcolemmal 'fuzzy' space and modulation of $[\mathrm{Ca} 2+] \mathrm{i}$ and contraction in cardiac myocytes. Cell Calcium 35(6): 603-612. doi: http://dx.doi.org/10.1016/j.ceca.2004.01.014.

Wang, X., and Abdel-Rahman, A.A. 2002. Estrogen modulation of eNOS activity and its association with caveolin-3 and calmodulin in rat hearts. American Journal of Physiology - Heart and Circulatory Physiology 282(6): H2309-H2315. doi: 10.1152/ajpheart.00772.2001.

Wetzel, G.T., and Klitzner, T.S. 1996. Developmental cardiac electrophysiology recent advances in cellular physiology. Cardiovascular Research 31(supp1): E52E60. doi: 10.1016/s0008-6363(95)00158-1.

Wu, Y., Colbran, R.J., and Anderson, M.E. 2001. Calmodulin kinase is a molecular switch for cardiac excitation -contraction coupling. Proceedings of the National Academy of Sciences 98(5): 2877-2881. doi: 10.1073/pnas.051449198.

Young, L.H., Ikeda, Y., and Lefer, A.M. 2001. Caveolin-1 peptide exerts cardioprotective effects in myocardial ischemia-reperfusion via nitric oxide 
mechanism. American Journal of Physiology - Heart and Circulatory Physiology 280(6): H2489-H2495.

Zhang, D., Shen, X., and Qi, X. 2016. Resting heart rate and all-cause and cardiovascular mortality in the general population: a meta-analysis. Canadian Medical Association Journal 188(3): E53-E63. doi: 10.1503/cmaj.150535.

Ziman, A.P., Gómez-Viquez, N.L., Bloch, R.J., and Lederer, W.J. 2010. ExcitationContraction Coupling Changes during Postnatal Cardiac Development. Journal of molecular and cellular cardiology 48(2): 379-386. doi: 10.1016/j.yjmcc.2009.09.016. 


\section{Figure legends}

Figure 1. Detection and distribution of STRN in cultured ventricular cardiomyocytes.

Panel A: PCR using STRN primers (ORF) and rat cardiac mRNA. Panel B: Western blot of protein lysate from cardiomyocyte and non-cardiomyocyte fractions. MHC (Myosin Heavy Chain), SMA (Smooth Muscle Actin). Panel C: immunofluorescence of STRN and alpha-actinin in cultured ventricular cardiomyocytes. The arrowheads highlight the expression of STRN at the sarcolemma.

\section{Figure 2. Developmental expression of cardiac striatin.}

Representative histograms of real time PCR (Panel A) and Western blot (Panel B) of STRN in the myocardium of $\mathrm{P} 1,2 \mathrm{~W}, 4 \mathrm{~W}$, and $13 \mathrm{~W}$ old rats. Panel C: Western blot showing the expression of STRN in right (R) and left (L) atria (A) and ventricles (V). GAPDH was used to confirm equal loading of proteins. * indicates $\mathrm{p}<0.05$ that was considered statistically significant when using unpaired t-test $(n=4)$.

Figure 3. The interaction of cardiac STRN with Cav-3 and CaM is calcium sensitive. Calmodulin $(\mathrm{CaM})$ pull down assay on cardiac protein extracts in the presence $(2 \mathrm{mM}$ $\mathrm{CaCl}_{2}$ ) or absence (EGTA) of calcium. Blots were probed with antibodies for Cav-3 and STRN. The input refers to $20 \mu \mathrm{g}$ of proteins in provenance of the same cardiac lysate used for the CaM pull down assay. 


\section{Figure 4. STRN regulates the spontaneous contraction rate of cultured}

\section{cardiomyocytes.}

Immunoblotting of STRN on protein lysates from cultured cardiomyocytes (Panel A) depleted from STRN (STRN-shRNA) or (Panel C) overexpressing STRN (RFP-STRN) or the reporter gene red fluorescent protein (RFP). GAPDH was used for control loading of proteins $(20 \mu \mathrm{g})$. The histograms in both Panel B and D represent the contraction rate, of cultured cardiomyocyte, expressed as percentage $(\%)$. ** indicates $\mathrm{p}<0.01$ and $* * *$ indicates $p<0.001$ using unpaired t-test of $n=4$ independent experiments.

\section{Figure 5. CaM pull down on cardiac lysates from cardiomyocytes with STRN OE or} KD.

Representative Western blot (Panel A and D) and calmodulin pull down assay (Panel B and E) using protein extracts from cardiomyocytes with STRN OE or KD, respectively. The reporter gene red fluorescent protein (RFP) served as a control for OE assay, while the Ctrl-shRNA for the KD experiments. Antibodies were used against STRN, Cav-3 and GAPDH (for equal loading of proteins). The input in Panel B \& E refers to $20 \mu \mathrm{g}$ of proteins from naïve cardiomyocytes used for OE or KD of STRN, respectively. Note the unchanged level of Cav-3 in cardiomyocytes with OE or KD of STRN, but the inversely proportional interaction of Cav-3 with CaM with the expression level of STRN. Panels C and $\mathrm{F}$ represent a paired t-test comparison of the cav-3 band (densitometry) in both $\mathrm{OE}$ and $\mathrm{KD}$ assays respectively. $\mathrm{P}<0.05$ was considered statistically significant in three independent experiments. 


\section{List of abbreviations}

$\left[\mathrm{Ca}^{2+}\right]_{\mathrm{i}}$ : intracellular calcium

E-C: excitation-contraction

HR: heart rate

STRN: striatin

AC: adenylyl cyclase

CaM: calmodulin

PP2A: protein phosphatase A2

Cav-1: caveolin-1

Cav-3: caveolin-3

$\mathrm{G}_{\alpha \mathrm{i}}:$ Gi alpha subunit

STRIPAK: striatin interacting phosphatases and kinases

SLMAP: sarcolemmal membrane associated protein

MEM: minimum essential medium

S-MEM: spinner minimum essential medium

FBS: fetal bovine serum

uM: micro molar

PSG: penicillin-streptomycin-glutamine

RIPA: radioimmunoprecipitation assay

PVDF: polyvinylidene difluoride

NFDM: nonfat dried milk

TBS-T: tris buffered saline with Tween

GAPDH: glyceraldehyde 3-phosphate dehydrogenase 
HRP: horseradish peroxidase

LTCC: L-type calcium channel

PCR: polymerase chain reaction

RNA: ribonucleic acid

mRNA: messenger ribonucleic acid

MOI: multiplicity of infection

RFP: red florescent protein

OE: overexpression

KD: knockdown

shRNA: short hairpin RNA

PBS: phosphate buffer saline

FCS: fetal calf serum

RT: room temperature

NCS: Normal Calf Serum

MHC: myosin heavy chain

SMA: smooth muscle actin

RA: right atrium

LA: left atrium

$\mathrm{RV}$ : right ventricle

LV: left ventricle

EGTA: ethylene glycol-bis( $\beta$-aminoethyl ether)-N,N,N',N'-tetraacetic acid

ARVC: arrhythmogenic right ventricle cardiomyopathy

SERCA: sarcoendoplasmic reticulum (SR) calcium transport ATPase 
CICR: calcium-induced calcium release

NCX: sodium-calcium exchanger 
A)

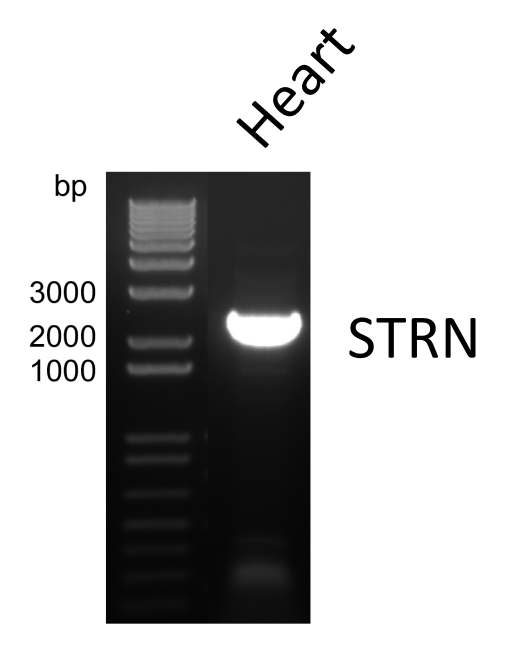

B)

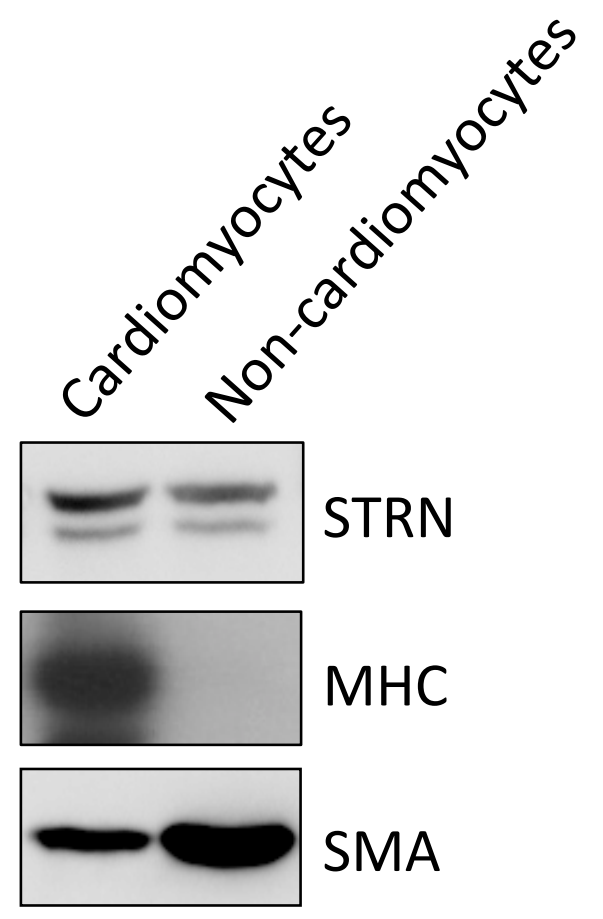

C)
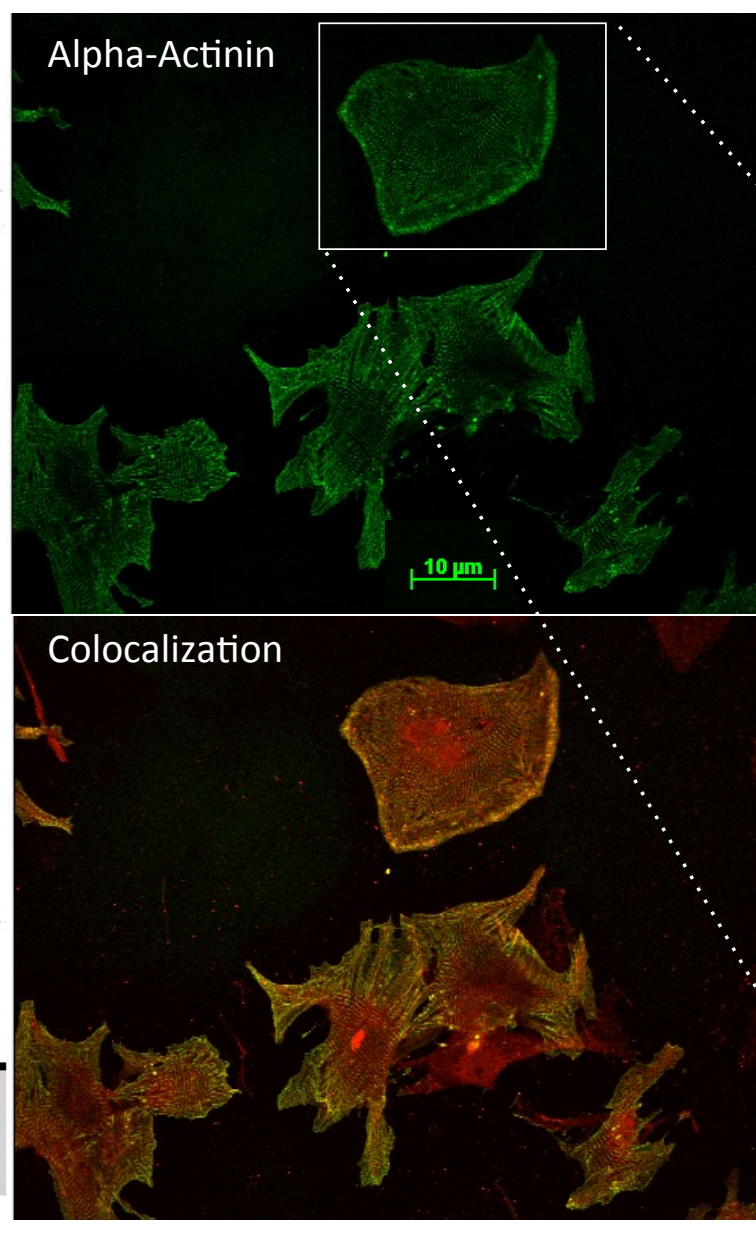

\section{Striatin}

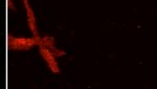

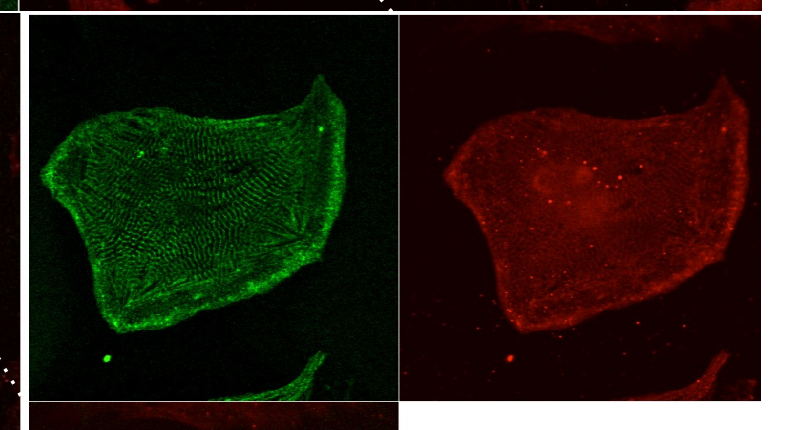


A)

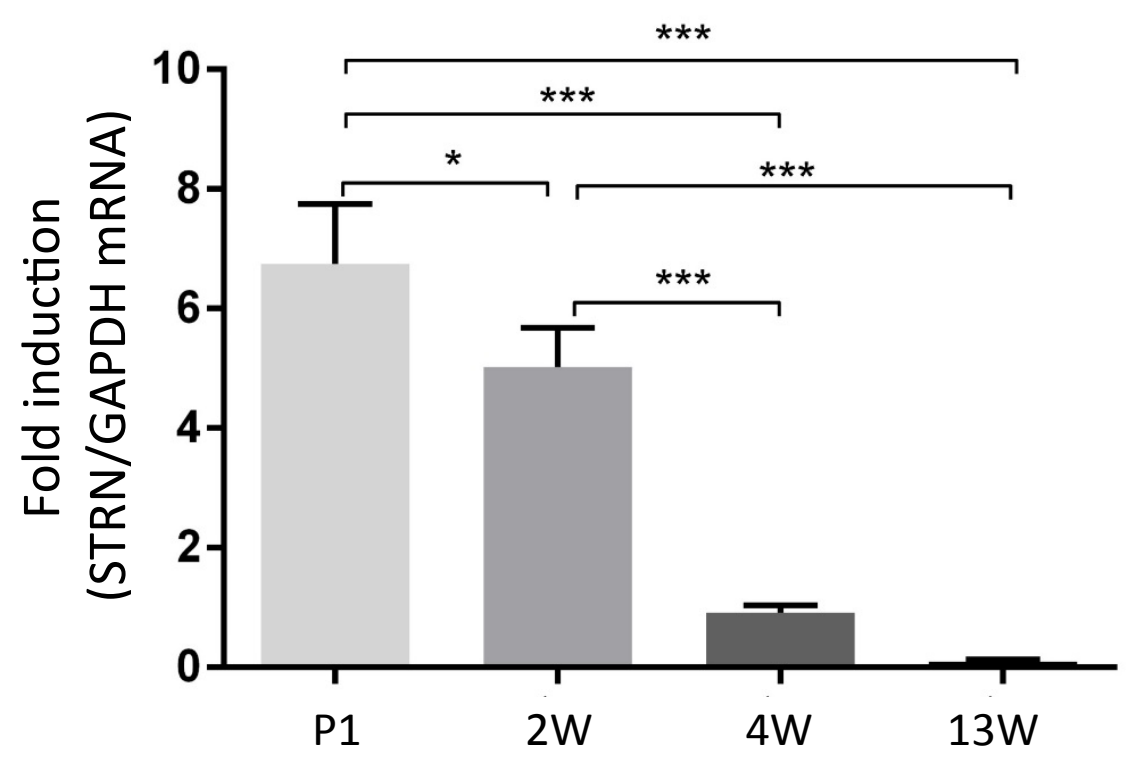

B)

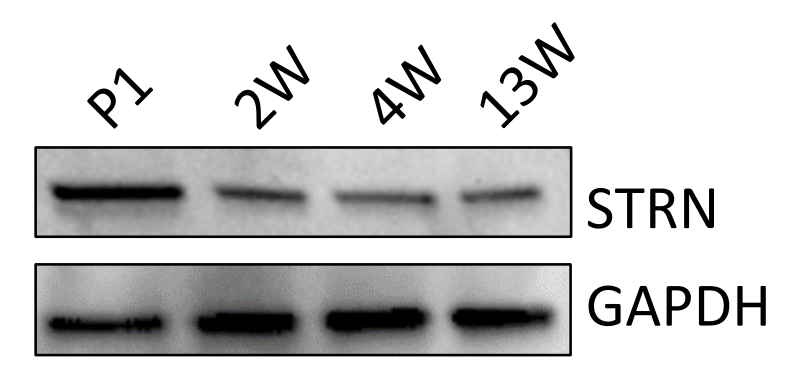

C)

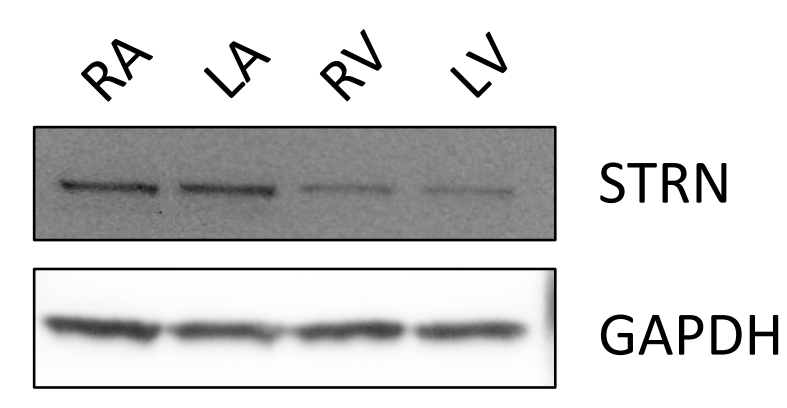




\section{Page 35 of 37
Figure 3}

\section{Canadian Journal of Physiology and Pharmacology}

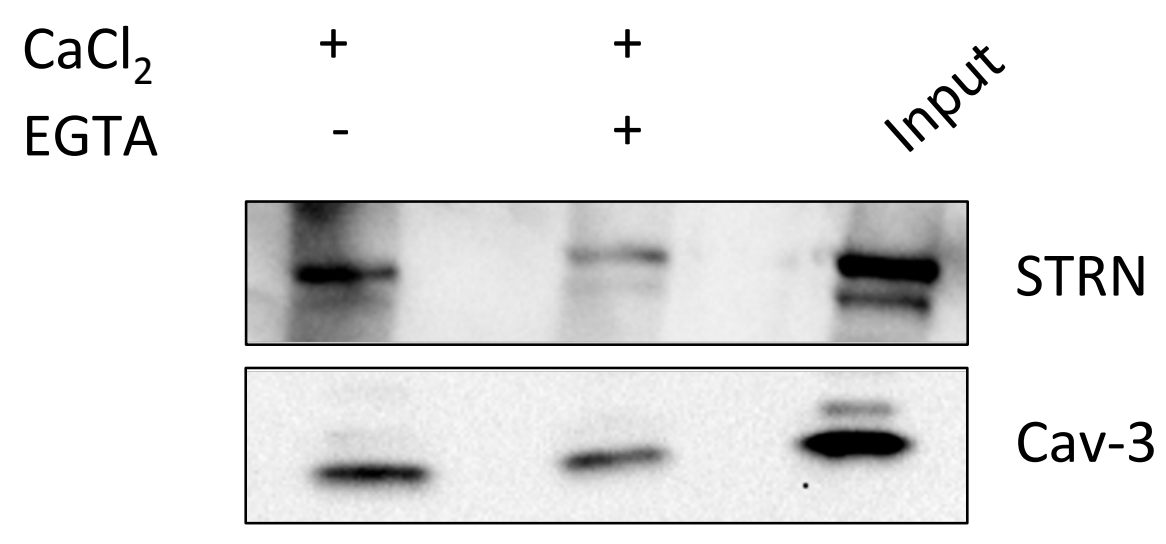

https://mc06.manuscriptcentral.com/cjpp-pubs 
A)

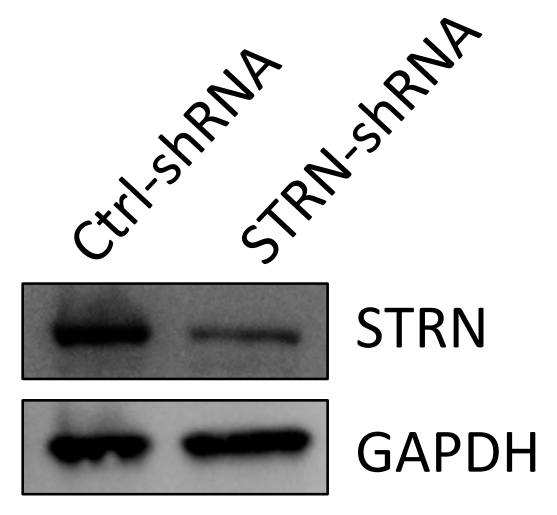

B)

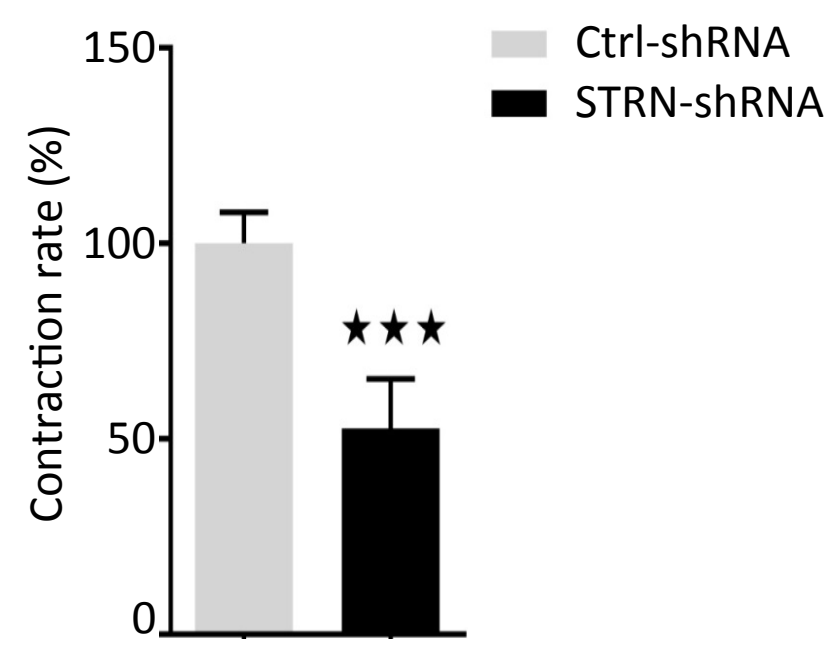

C)

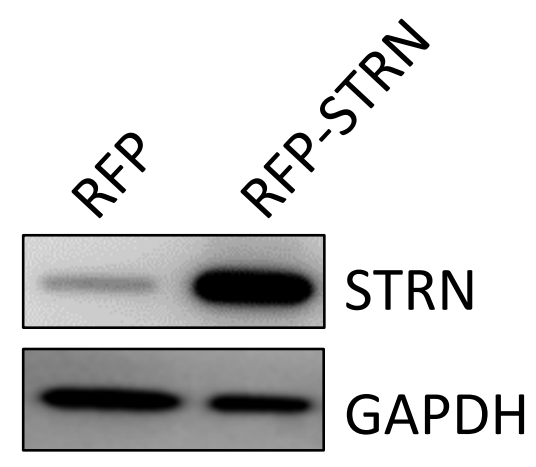

D)

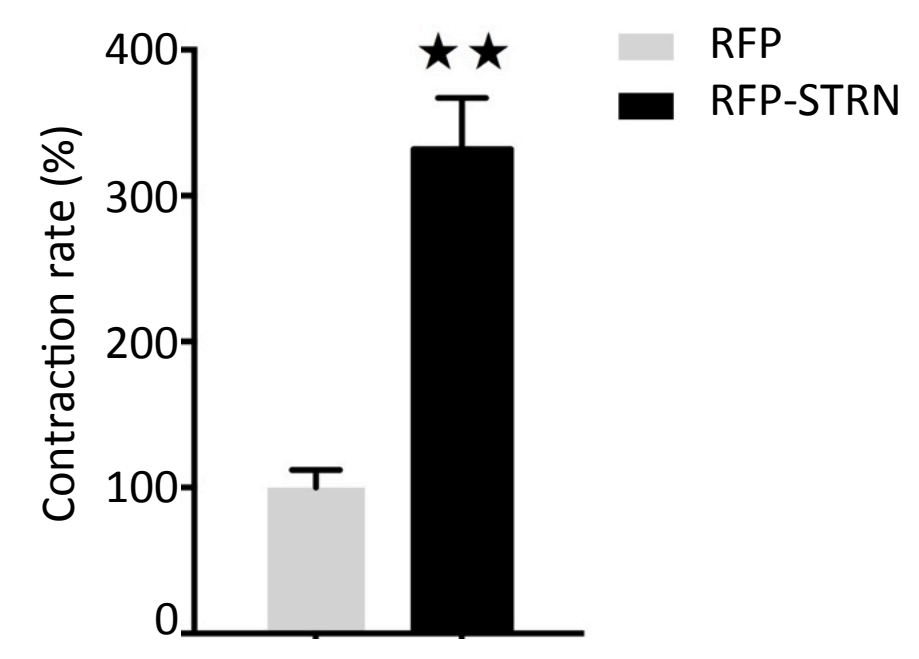


A)

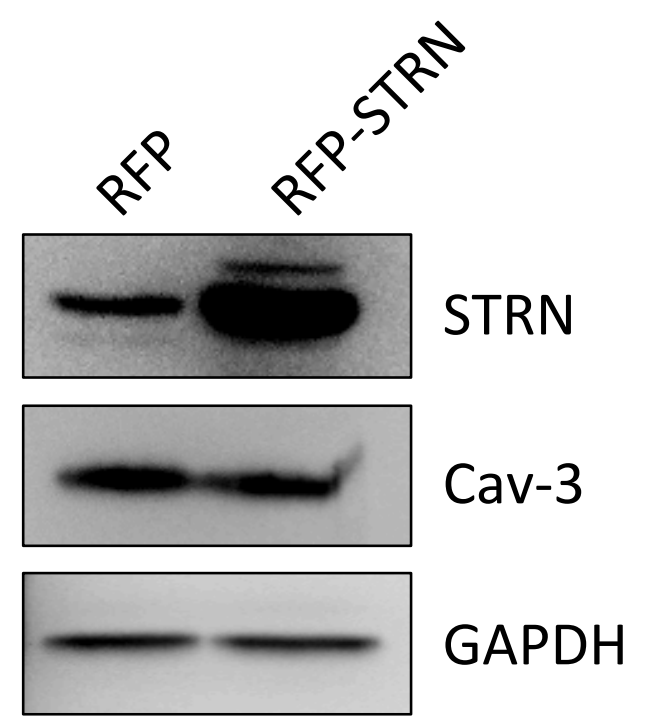

D)

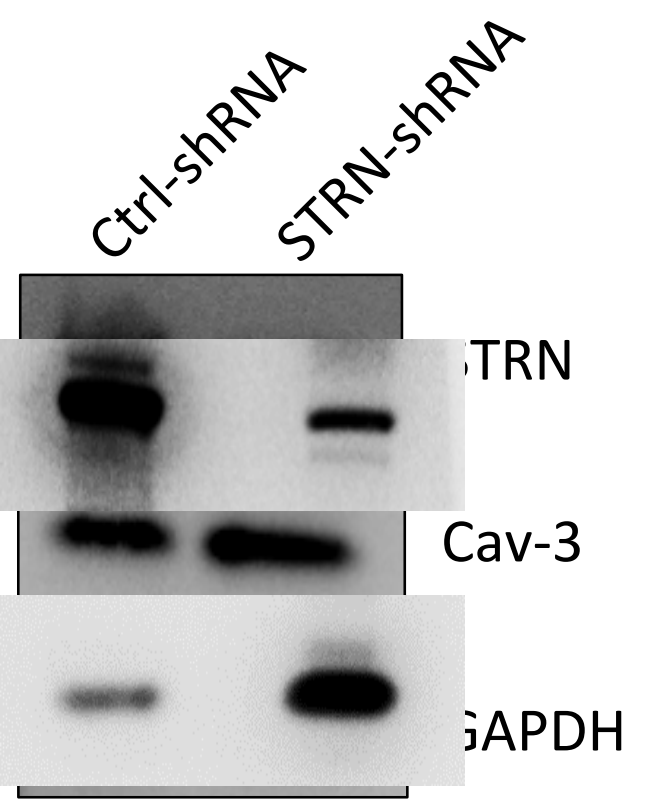

B)

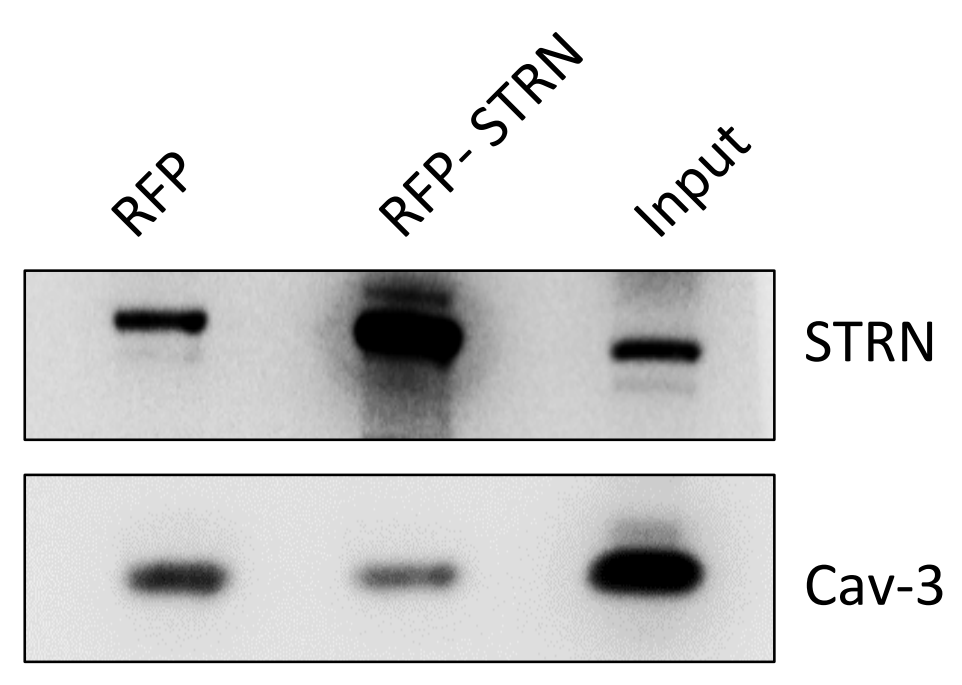

E)

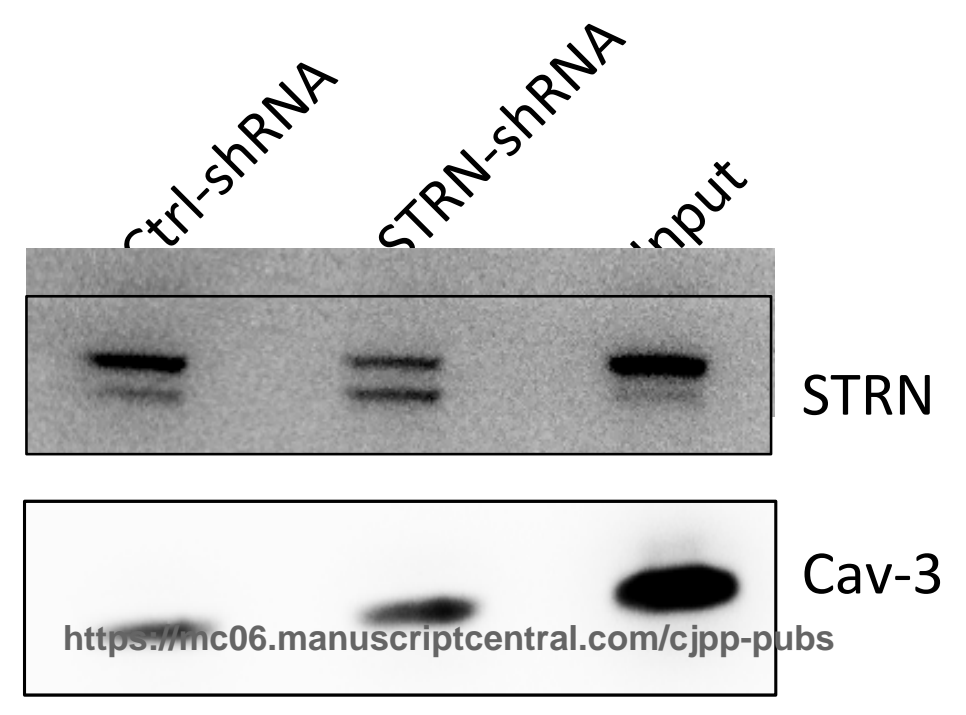

C)

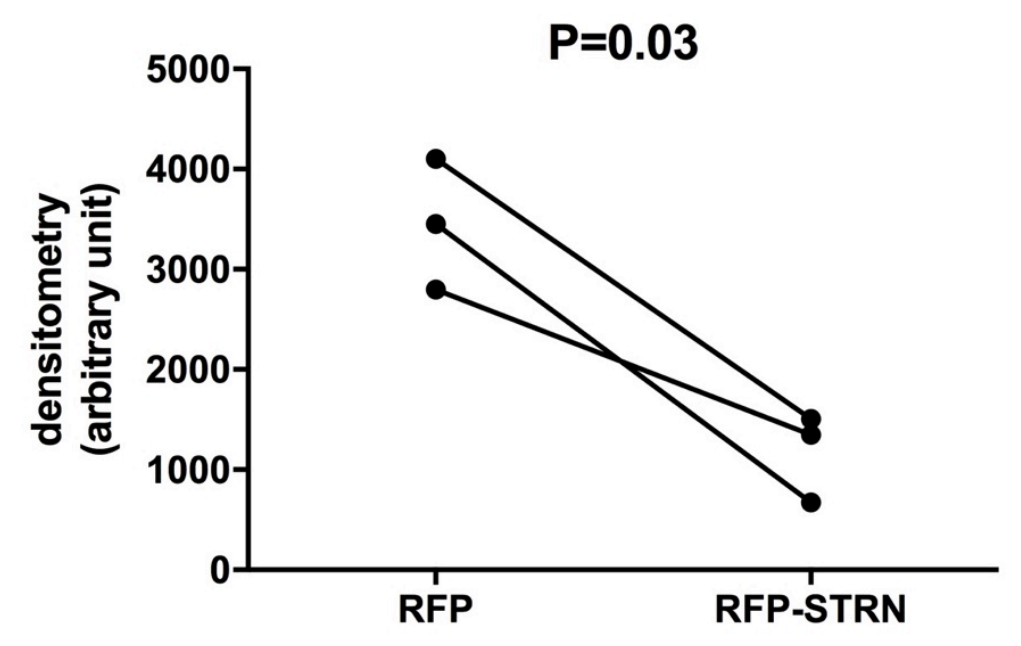

F)

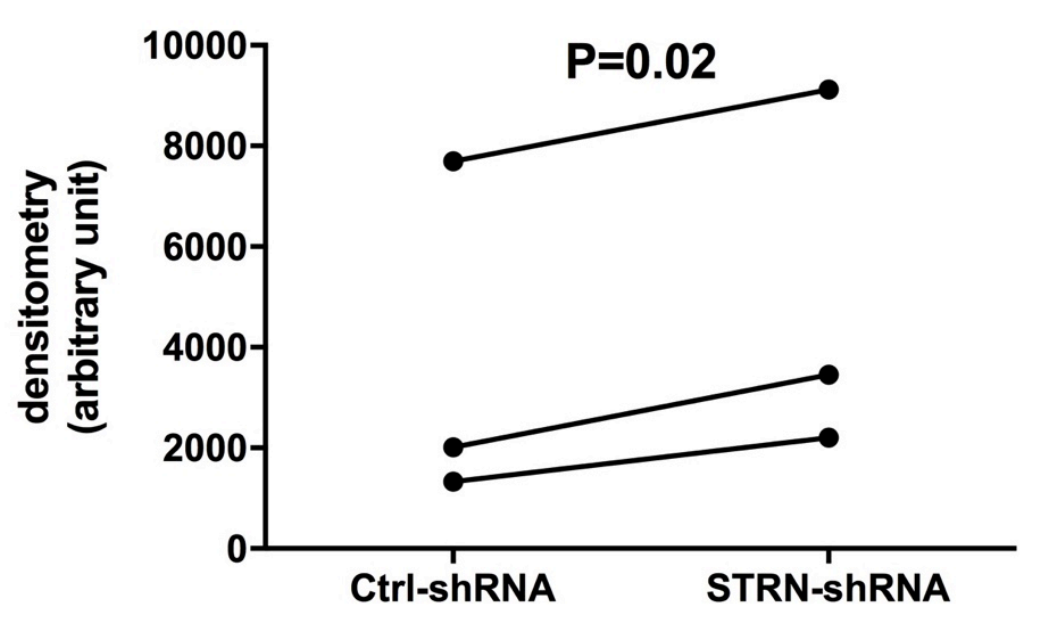

